\title{
New advances in EMG control methods of anthropomorphic prosthetic hand
}

\author{
WANG YiXuan ${ }^{1 \dagger}$, LIU He ${ }^{1 \dagger}$, LENG DongLiang ${ }^{2}$, GAO Wei $^{3 *}$, CAI MaoLin ${ }^{1}$, XU WeiQing ${ }^{1}$, \\ ZHANG Xiaohua Douglas ${ }^{2} \&$ SHI Yan ${ }^{1,2,3,4^{*}}$ \\ ${ }^{1}$ School of Automation Science and Electrical Engineering, Beihang University, Beijing 100191, China; \\ ${ }^{2}$ Faculty of Health Sciences, University of Macao, Taipa, Macao; \\ ${ }^{3}$ The State Key Laboratory of Fluid Power Transmission and Control, Zhejiang University, Hangzhou 310058, China: \\ ${ }^{4}$ Beijing Anzhen Hospital Capital Medical University, Beijing 100029, China.
}

Received August 7, 2017; accepted October 9, 2017; published online November 14, 2017 Citation: Wang Y X, Liu H, Leng D L, et al. New advances in EMG control methods of anthropomorphic prosthetic hand. Sci China Tech Sci, 2017, 60:
1978-1979, doi: 10.1007/s11431-017-9159-3

Prosthetic hands are becoming more and more popular among people without a hand and it is able to perform certain manual operations in function. In biology, action potentials trigger muscle contractions, and surface electromyography (sEMG) signals are the sum of action potentials under the skin exposed by the electrodes (via sebum and solution conduction). Myoelectric control is a technique that relies on extracting information contained in SEMG signals to determine muscle contractions and then control peripheral devices [1-3]. However, how to collect sEMG signals effectively is the first difficult problem for scholars [4] because of weakness, randomness and low signal-to-noise ratio of the sEMG signals. Multi DOF prosthetic hands have high flexibility and then more control parameters are needed. Therefore, the key techniques of EMG control of anthropomorphic prosthetic hand are extracting appropriate control parameters and studying real-time and reliable control algorithms.

At present, single-degree-of-freedom prosthetic hands are one of the mainstream of commercial prosthesis markets. With mature EMG signal processing and control system, the performance of them is stable, but the flexibility is not enough. There are also some multi DOF prosthetic hands

\footnotetext{
$\uparrow$ These authors contributed equally to this work

* Corresponding authors (email: gaow13975@163.com; shiyan@buaa.edu.cn)
}

that have been commercialized, such as the I-limb prosthesis introduced by Touch Bionics (Scotland) with 5 independent fingers, 24 degrees of freedom. But it's so expensive that ordinary people can't afford it. researchers have proposed a variety of myoelectric control schemes, including threshold based decision, amplitude-based coding method, hierarchical control decision, etc. [5,6]. The thresholding method is that an EMG signal caused by muscle contraction is firstly corrected, filtered and modulated, that then its peak value is compared with the threshold(s), and that finally two or three states of output correspond to the prosthetic hand to relax, bending or stretching, respectively. The scheme is so simple and reliable that it is the control method for a single-degree-of-freedom prosthetic hand. However, due to less information given by each electrode, it is not suitable for the control of a multi DOF prosthetic hand.

Like the thresholding decision, in the amplitude-based coding scheme [7], the amplitudes of sEMG collected by differential electrodes are divided into three states, and the artificial hand motion commands are determined by encoding outputs according to time sequence. This decision is simple, whereas requires a lot of training, with low real-time and inflexible control. About the hierarchical control decision, the hand grasp modes of muscle stump are recognized by using advanced signal modulation and pattern recognition al- 
gorithms. The underlying controllers implement the stable grasp of the object by the artificial hand. This kind of structure makes it possible to control the multi-degree-of-freedom hands with fewer electrodes. However, the prosthetic grasping mode based on the sensor information can sometimes not accurately reflect the control intention.

Pattern recognition technology is a new method for myoelectric control. The sEMG signals are captured by electrodes attached to the body surface and they must be filtered, enlarged and modulated in order to correctly monitor action potential caused by muscle contraction [8,9]. Next work is extracting proper features from effective sEMG signals and input the features into the classifier to make judgments about all kinds of movement patterns. Finally, the results are inputted into the bottom controllers, which help artificial hands to complete similar actions.

The features used to calculate the original EMG signals can be divided into three categories [10]: the first is the time-domain characteristics, including the EMG integral, Root Mean Square (RMS), zero crossing points, etc., and the time-domain features have good classification characteristics. The second one is frequency domain characteristics, containing average frequency, median frequency, peak frequency, average power, and so on. Frequency domain characteristics are mainly used to study muscle fatigue and movement unit recruitment. The third is time-frequency domain characteristics, such as wavelet transform, short-time Fourier transform, etc., which can simultaneously analyze the signals in the time domain and frequency domain, but the calculation is more troublesome. The classifier [11] for EMG signals requires strong generalization ability and fast training and recognition ability. At present, BP neural network has become a mature pattern classifier in the field of EMG pattern recognition [12]. Besides, support vector machine (SVM) is also used in this field [13]. They all got an ideal correct rate of recognition.

Myoelectric control based on pattern recognition has a more than $90 \%$ recognition rate of multiple motion modes (flexion, extension, rotation, etc.). Therefore, the control of multi-degree of freedom hands is more intuitive and flexible. In a word, myoelectric control based on pattern recognition is the future development direction of the control of multivariant prosthetic hands, because with the rapid development of pattern recognition algorithms, the recognition accuracy of myoelectric control system becomes higher, the control effect is more intuitive, real-time is also better. The study can be referred to the development of diagnosis and rehabilitation technologies of respiratory [14-19].

This work was supported by the National Natural Science Foundation of China (Grant No. 51575020), the National Key Technology R\&D Program
(Grant No. 2015BAI02B00) and Open Foundation of the State Key Laboratory of Fluid Power and Mechatronic Systems.

1 Ma J, Thakor N V, Matsuno F. Hand and wrist movement control of myoelectric prosthesis based on synergy. IEEE Trans Human-Mach Syst, 2015, 45: 74-83

2 Nadi M, Midha R. Editorial: Myoelectric functional hand prosthesis for total brachial plexus injury. J Neurosurgery, 2011, 127: $1160-1162$

3 Shi Y, Wang Y, Cai M, et al. An aviation oxygen supply system based on a mechanical ventilation model. Chin J Aeronautics, 2017

4 Smith L H, Kuiken T A, Hargrove L J. Evaluation of linear regression simultaneous myoelectric control using intramuscular EMG. IEEE Trans Biomed Eng, 2016, 63: 737-746

5 Parker P, Englehart K, Hudgins B. Myoelectric signal processing for control of powered limb prostheses. J Electromyogr Kinesiol, 2006, 16: $541-548$

6 Reischl M, Mikut R, Pylatiuk C, et al. Steuerungs- und Signalverarbeitungskonzepte für eine multifunktionale Handprothese (Control and Signal Processing Concepts for a Multifunctional Hand Prosthesis). at-Automatisierungstechnik, 2002, 50

7 Pons J L, Ceres R, Rocon E, et al. Virtual reality training and EMG control of the MANUS hand prosthesis. Robotica, 1999, 23: 311-317

8 Zecca M, Micera S, Carrozza M C, et al. Control of multifunctional prosthetic hands by processing the electromyographic signal. Crit Rev Biomed Eng, 2002, 30: 459-485

9 Reaz M B I, Hussain M S, Mohd-Yasin F. Techniques of EMG signal analysis: Detection, processing, classification and applications. Biol Proced Online, 2006, 8: 11-35

10 Huang Y, Englehart K B, Hudgins B, et al. A Gaussian mixture model based classification scheme for myoelectric control of powered upper limb prostheses. IEEE Trans Biomed Eng, 2005, 52: 1801-1811

11 Oskoei M A, Hu H. Adaptive myoelectric human-machine interface for video games. In: International Conference on Mechatronics and Automation IEEE, 2009. 1015-1020

12 Liang F, Li C, Gao Y, et al. Study on pattern recognition of hand motion modes based on wavelet packet and SVM. 2014, 462: 180-188

13 Chen X, Zhang D, Zhu X. Application of a self-enhancing classification method to electromyography pattern recognition for multifunctional prosthesis control. J Neuroeng Rehabil, 2013, 10: 44

14 Niu J, Shi Y, Cai M, et al. Detection of sputum by interpreting the time-frequency distribution of respiratory sound signal using image processing techniques. Bioinformatics, 2017, doi: 10.1093/bioinformatics/btx65215

15 Niu J L, Shi Y, Cao Z X, et al. Study on air flow dynamic characteristic of mechanical ventilation of a lung simulator. Sci China Tech Sci, 2017, 60: 243-250

16 Ren S, Shi Y, Cai M, et al. Influence of secretion on airflow dynamics of mechanical ventilated respiratory system. IEEE/ACM Trans Comput Biol Bioinf, 2017, 1-1

17 Ren S, Cai M, Shi Y, et al. Influence of bronchial diameter change on the airflow dynamics Based on a pressure-controlled ventilation system. Int J Numer Meth Biomed Engng, 2017, e2929

18 Shi Y, Zhang B, Cai M, et al. Coupling effect of double lungs on a VCV ventilator with automatic secretion clearance function. IEEE/ACM Trans Comput Biol Bioinf, 2017, 99: 1

19 Shi Y, Zhang B, Cai M, et al. Numerical simulation of volume-controlled mechanical ventilated respiratory system with 2 different lungs. Int J Numer Meth Biomed Engng, 2017, 33: e2852 